\title{
THE EXPLOSION OF A ROTATING MAGNETIZED STAR
}

\section{AS A SUPERNOVA}

(Abstract)

\author{
G. S. BISNOVATYI-KOGAN
}

Institute of Applied Mathematics, USSR Academy of Sciences, Moscow, U.S.S.R.

It is easy to show that a moderately rotating star, which is contracted to the size of a neutron star, will rotate very rapidly if angular momentum is conserved. In fact, if on the main sequence the equatorial rotation speed is $10 \mathrm{~km} \mathrm{~s}^{-1}$ at $R_{\mathrm{eq}}=10^{11} \mathrm{~cm}$, then, after the contraction to $R=10^{6} \mathrm{~cm}, v=10\left(R_{\mathrm{eq}} / R\right)>c$; of course the relativistic formula is necessary in order to obtain $v<c$. Thus, when rotation has rather small importance in early stages of evolution, it may play a decisive role in late stages, particularly in a supernova explosion.

The existence of pulsars shows that magnetic fields in neutron stars are very large $\left(\sim 10^{12} \mathrm{G}\right)$. In Bisnovatyi-Kogan (1970) a mechanism of supernova explosion was proposed in which rotation was the main source of energy and the magnetic field provided the mechanism for its release. After contraction from the rapidy rotating pre-supernova stage, a differentially rotating disc-like configuration is formed, with a rapidly rotating neutron core. The transfer of angular momentum from the core to the envelope due to magnetic connection leads to the formation of a compressional wave in the envelope, which propagating in a medium of decreasing density transforms into a shock wave which appears as a supernova explosion.

A numerical calculation has confirmed this qualitative picture. Although the calculation assumes cylindrical geometry, it seems clear that in the more realistic spherical geometry the magnetorotational mechanism is important. It seems to me that the mechanism of supernova explosion due to pulsar-like emission will, in contrast, not work, because the high density plasma around the neutron star will screen out slow waves.

\section{Reference}

Bisnovatyi-Kogan, G. S.: 1970, Astron. Zh. Akad. Nauk SSSR 47, 813. 